\section{6th Annual Conference on Atomic Spectroscopy}

Berlin-West, DDR, 16-19 July 1974

This year a group of colleagues from the Technische Universität in Berlin organized the sixth conference in the series of annual conferences on atomic spectroscopy that have been started on the initiative of the European Group for Atomic Spectroscopy (EGAS), nowadays a section of the Atomic Physics Division of EPS.

The date had been settled in concert with the organizers of the Fourth International Conference on Atomic Physics in Heidelberg. Consequently the conferences were held in two consecutive weeks.

About 200 participants assembled in Berlin to hear, as usual, a few invited papers and a large number of short contributed papers, read in two parallel sessions.

Term analysis of atomic spectra accounted for nearly one third of the more than 70 contributed papers; resonance fluorescence and optical pumping, laser investigations, beamfoil techniques each occupied one sixth of the time, papers on optical hyperfine structure and on impact phenomena accounted for the rest.

In his opening lecture Professor A. Kastler gave a review of "50 years Hanle effect", Professor Hanle, attending this lecture.

The large contribution of term analysis was reflected in the other invited papers: Professor B. Judd from Johns Hopkins University, Baltimore, asked in connection with his lecture "Theoretical techniques for many-electron spectra" for more experimental data on only one electron configuration, namely either $\mathrm{f}^{4}$ or $\mathrm{f}^{10}$. On the other hand Dr. W.L. Wiese from N.B.S., Washington, demonstrated in his lecture "Atomic transition probabilities - present status and needs" the need to fill the many "gaps" in term analysis of highly ionized atoms. In both cases experimentalists are confronted with the problem how to control excitation of the atomic levels concerned. Atomic levels above the ionization limit were the subject of Dr. Connerade from Imperial College, London in his lecture "Inner shell effects in atomic spectra".

Apart from scientific activities a General Assembly of EGAS was held. It was announced that the next (7th) Annual Conference will be held in Grenoble.

\section{E. Meinders, Amsterdam}

\section{announcing a new journal from North-Holland Publishing Co:}

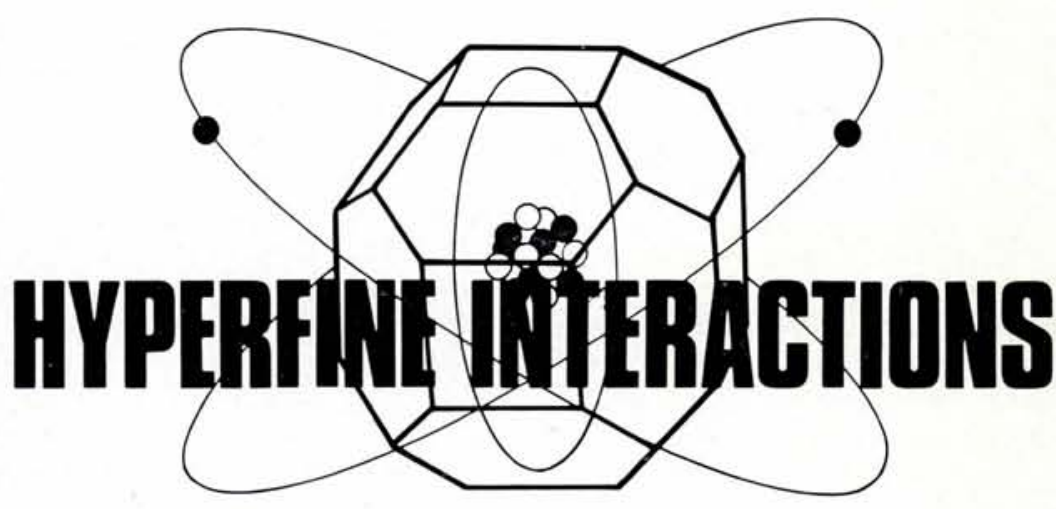

Editors:

H. DE WAARD, Physics Laboratory, University of Groningen, Groningen. The Netherlands

B. DEUTCH, Institute of Physics, University of Aarhus, Aarhus, Denmark

\section{Scope}

The journal is concerned with research in the border regions of solidstate, atomic, and nuclear physics. Although the title and subject HYPERFINE INTERACTIONS is one of the best examples of such a topic, other studies which utilize the modern techniques of atomic and nuclear physics may fall within the domain of the journal. The main concern is the understanding of border region physics, no matter what are the disguised variegated forms in which it appears.

The hyperfine interaction Hamiltonian is the logical basis for a large part of the subject matter to which this journal will be devoted because it contains products of atomic and nuclear quantities. In condensed matter, the Hamiltonian even spans solid-state physics. Hence such fields as: perturbed angular correlations, nuclear magnetic resonance, electric quadrupole resonance, beam-foil spectroscopy, nuclear orientation, and Mössbauer effect studies fall immediately within the journals domain. In addition, any atomic or solid-state studies via hyperfine spectroscopy (utilizing mesons, gamma rays, electrons, etc.) are within the scope. It can include problems related to solid, liquid and gaseous states as well as studies with biological material.

The title HYPERFINE INTERACTIONS is not meant to prevent contributions that do not directly involve such interactions from reaching the journal. For example, the journal is receptive to particle-beam studies which also explore the border physics region but do not necessarily utilize hyperfine effects. Under this category lie channeling. nuclear lifetimes studied via channeling and implantation phenomena. In fact, studies which incorporate one of the disciplines of physics: solid-state, atomic or nuclear physics to study another would be generally acceptable.

\section{Advisory editorial board}

An editorial board is being formed and to-date the following scientists already accepted our invitation: B. Herskind, Copenhagen; E. Matthias, Berlin; J. A. Davies, Chalk River; N. Stone, Oxford E. Bodenstedt, Bonn; G. Goldring, Rehovat; V. I. Goldanski, Moscow; T. Yamazaki, Tokyo:

\section{Invitation to authors}

The editors cordially invite colleagues to submit papers for publication within the scope of the journal. It should be realised that the refeering criteria will be high. Guidelines for preparing the manuscripts are available from the editors and publisher. Contributions should be sent to B. Deutsch, University of Aarhus.

\section{Subscription information}

The journal will be published in volumes of 6 issues; each issue will contain some 100 pages, first issue is scheduled to appear in spring 1975; In principle the journal will appear bimonthly. Subscription price: US $\$ 43.95$ Dfl.110.00 per volume, postage included. Specimen copies will be supplied by the publisher. 